\title{
Optimization of Robot Inverse Kinematics based on the Multi-joint-drive Speed Coordinated Energy Consumption Method
}

\author{
Enlei Chen ${ }^{a}$ and Changjun Liu ${ }^{b}$
}

\author{
School of Mechanical and Power Engineering, East China University of Science and Technology, \\ Shanghai 200237, China \\ a1272375234@qq.com, b13162218709@163.com
}

\begin{abstract}
For the cascade five-degree-of-freedom inchworm-imitating bipedal climbing robot body mechanism, multiple kinematic inverse solutions maybe generated due to redundancy in the $\mathrm{D}-\mathrm{H}$ kinematics modeling process. Therefore, an optimal kinematic inverse solution selection method based on energy consumption is proposed. This method studies the driving load or robot joints first, and works out the static load energy consumption function between gaits with the static load simplification method. Then, it carries out coordinated control of the cascade robot joint driving speed to obtain the joint's rotational angular velocity function, which is substituted into the parameter to work out the energy consumption corresponding to the multiple inverse solutions. The optimal inverse kinematics solution is screened with minimal energy consumption. Finally, the rationality and effectiveness of the inverse kinematics optimization solution method are verified through MATLAB simulation analysis.
\end{abstract}

Keywords: Inverse kinematics, energy consumption analysis, multi-joint-drive speed coordinated (MSC), MATLAB simulation

\section{Introduction}

Inchworm, a soft-bodied insect in nature, is taken as the bionic object of this subject, which designs a cascade robot system of five revolute joints. As shown in Fig.1(a), $\mathrm{I}_{1}-\mathrm{I}_{2}$ are two coaxial revolute joints at both ends of the cascade mechanism, being available for $\pm 180^{\circ}$ rotation. $T_{1}-T_{2}$ and $T_{3}$ are three rotation swing joints evenly distributed in the middle of the mechanism; absorbing feet of the robot $S_{1-} S_{2}$ are located at both ends of the mechanism. The overall structure is symmetrical with the difficulty of motion control reduced[1, 2].

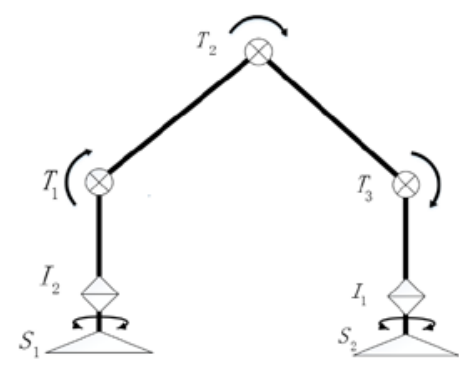

(a)

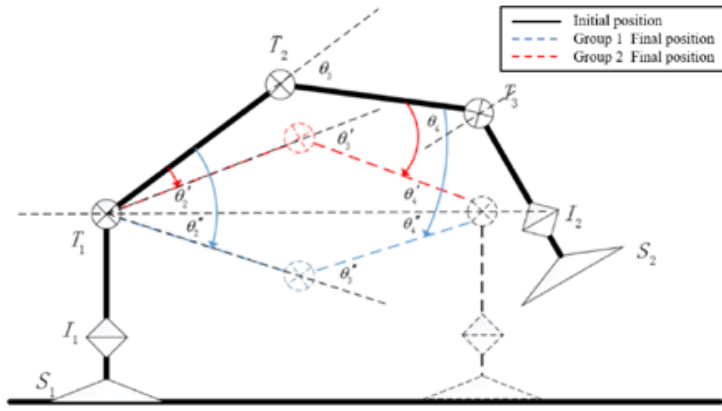

(b)

Fig.1 Bio-Inchworm wall climbing robot, (a) Body configuration; (b) Inverse kinematics solution

The Denavit-Hartenberg method, a common method for calculating robot kinematical function with matrix recursive expressions, is frequently used for robot kinematical calculation of various structures for the freedom from considering the robot structure and the configuration sequence [3]. Therefore, the robot kinematics model is built based on D-H method to solve the kinematics problems. However, since multiple sets of inverse solutions may be generated for the robot inverse kinematics calculation due to the redundancy of cascade robot with the determination of the inter-gait movement process of robot and the preparation of control programs hindered is shown in Fig.1(b). The multiple sets of inverse kinematics solutions shall be optimized and selected. 


\section{Robotic Energy Consumption Analysis}

\subsection{Multi-joint static load simplification}

Due to the Bio-Inchworm robot, the robot servo system of each joint drive steadily and slowly rotates through the target rotation angle. Regardless of the thermal energy loss of the joint drive motor, only the joint motor resists the torsion of its own potential energy during the robot motion process, and it is used as the energy consumption of the motor for the effective load generated by the motion process [4].

As shown in Fig.2, the quality of the joint drive, reducer, and other components of the robot body structure is reduced to the equivalent mass $M_{m 1} \sim M_{m 5}$ acting on the joint rotation center. Secondly, the masses of the robot arms and the electromagnetic adsorption feet between the joints are also reduced to equal mass parameters $M_{L 1} \sim M_{L 6}, M_{S 1}$, and $M_{S 2}$ that act on the midpoint of the arm or the center of the foot.

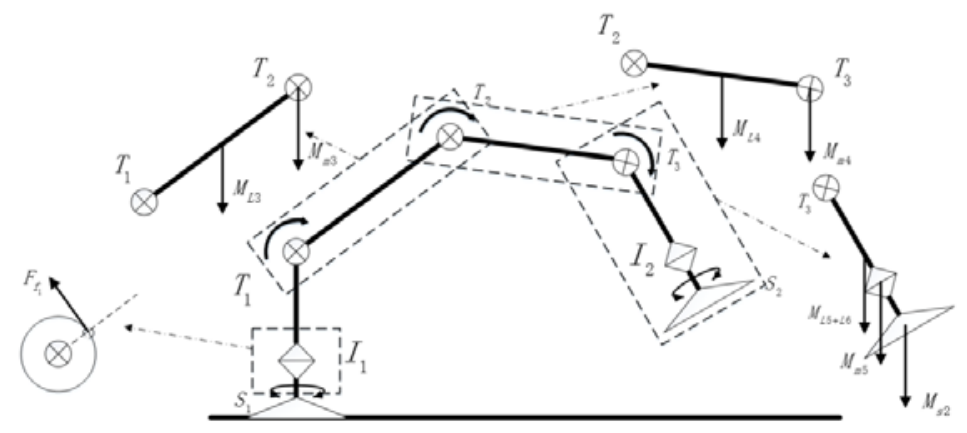

Fig.2 Multi-joint static load simplification

In power formula $W=F \cdot S \cos \theta$ energy calculation basis for the analysis of simulation robot joint driving Bio-Inchworm static load. To obtain $\mathrm{T}_{1}, \mathrm{~T}_{2}$ and $\mathrm{T}_{3}$ energy consumption expression of static load:

$$
\begin{aligned}
W_{T_{3}} & =\int_{0}^{T}\left[\left(M_{\mathrm{s}_{2}}+\frac{M_{L_{5}+L_{6}}}{2}\right)\left(L_{5}+L_{6}\right)+M_{m_{5}} L_{5} l g \cdot \sin \theta_{T_{3}} \cdot \omega_{T_{3}}(t) d t\right. \\
W_{T_{2}} & =\int_{0}^{T}\left[\left(M_{\mathrm{s}_{2}}+\frac{M_{L_{5}+L_{6}}}{2}\right)\left(L_{5}+L_{6}\right)+M_{m_{5}} L_{5} l g \sin \theta_{T_{3}} \cdot \omega_{T_{2}}(t) d t\right. \\
& +\int_{0}^{T}\left(M_{\mathrm{s}_{2}}+M_{\mathrm{m}_{5}}+M_{\mathrm{m}_{4}}+M_{L_{5}+L_{6}}+\frac{M_{\mathrm{L}_{4}}}{2}\right) g L_{4} \cos \theta_{T_{2}} \cdot \omega_{T_{2}}(t) d t \\
W_{T_{1}}= & \int_{0}^{T}\left[\left(M_{\mathrm{s}_{2}}+\frac{M_{L_{5}+L_{6}}}{2}\right)\left(L_{5}+L_{6}\right)+M_{m_{5}} L_{5} l g \cdot \sin \theta_{T_{3}} g \omega_{T_{1}}(t) d t\right. \\
& +\int_{0}^{T}\left(M_{s_{2}}+M_{m_{5}}+M_{m_{4}}+M_{L_{5}+L_{6}}+\frac{M_{L_{4}}}{2}\right) g L_{4} \cdot \cos \theta_{T_{2}} \cdot \omega_{T_{1}}(t) d t \\
& +\int_{0}^{T}\left(M_{s_{2}}+M_{m_{5}}+M_{m_{4}}+M_{m_{3}}+M_{L_{5}+L_{6}}+M_{L_{4}}+\frac{M_{L_{3}}}{2}\right) g L_{3} \cdot \cos \theta_{T_{1}} \cdot \omega_{T_{1}}(t) d t \\
& W_{A}=W_{T_{1}}+W_{T_{2}}+W_{T_{3}}
\end{aligned}
$$

The static load energy consumption during the movement of the joints $T_{1}, T_{2}$, and $T_{3}$ can be accumulated to obtain the total static load energy consumption $W_{A}$ during the gait movement. Therefore, it is necessary to obtain the rotation speed function $\omega_{T_{1}}(t), \omega_{T_{2}}(t), \omega_{T_{3}}(t)$ and the movement time $t$ of each joint driver unit.

\subsection{Multi-joint-drive speed coordinated (MSC) method}

In order to obtain the joint movement speed curve, support the robot movement energy consumption calculation. To achieve multi-joint coordinated control of robots, it is necessary to find the angle of movement of each joint according to the joint motion trajectory of the robot.. Multi-joint-drive speed coordinated control method can be divided into two parts: speed-equal proportional coordination (SPC) and speed-equal time coordination (STC).

(1) Speed-equal proportional coordination (SPC) 
The maximum value of the joint rotation between the gaits of the robot is used as a reference value, and the acceleration time parameter is set according to the trapezoidal lift speed algorithm. It can get the speed curve of the joint drive to complete fixed-length motion at the same time [5].

As shown in Fig. 3(a), $T_{\text {std }}$ is set as the time when the robot moves from one attitude to another, and the servo motor drive unit accelerates during the time period $\left[0, t_{1}\right]$; the time period $\left[t_{1}, t_{2}\right]$ moves at a constant speed and the time period $\left[t_{2}, T\right]$ decelerates. When $t_{1}=T / 3, t_{2}=2 T / 3$ the energy consumption value of the trapezoidal speed-up algorithm is the smallest [6]. Finally get the joint rotation speed standard function curve:

$$
\omega_{G_{\mathrm{i}}}(t)=\left\{\begin{array}{cc}
\frac{9 \theta_{\text {std }}}{2 T_{\text {std }}{ }^{2} \cdot t} & 0 \leq t \leq \frac{T_{G_{i}}}{3} \\
\frac{3 \theta_{\text {std }}}{2 T_{\text {std }}} \cdot T_{G_{i}} & \frac{T_{G_{i}}}{3}<t \leq \frac{2 T_{G_{i}}}{3} \quad i=1,2 \\
-\frac{9 \theta_{\text {std }}}{2 T_{\text {std }}{ }^{2}} \cdot t+\frac{9 \theta_{\text {std }}}{2 T_{\text {std }}{ }^{2}} \cdot T_{G_{i}} & \frac{2 T_{G_{i}}}{3}<t \leq T_{G_{i}} \\
T_{G_{i}}=\sqrt{\frac{\Delta \theta_{G_{i} \max } \cdot T_{s t d}}{\theta_{\text {std }}}}
\end{array}\right.
$$

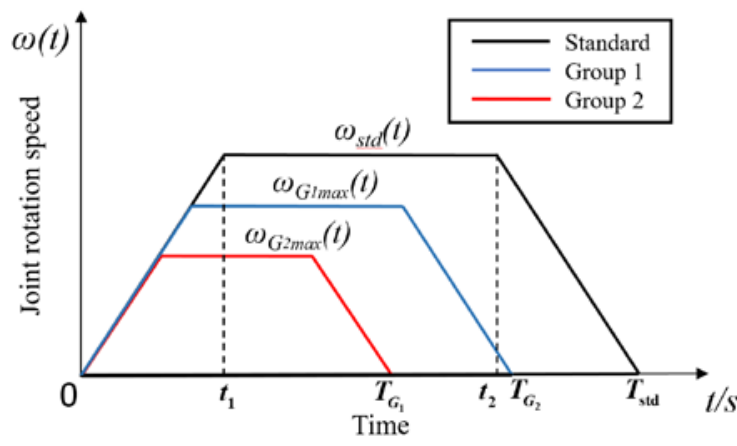

(a)

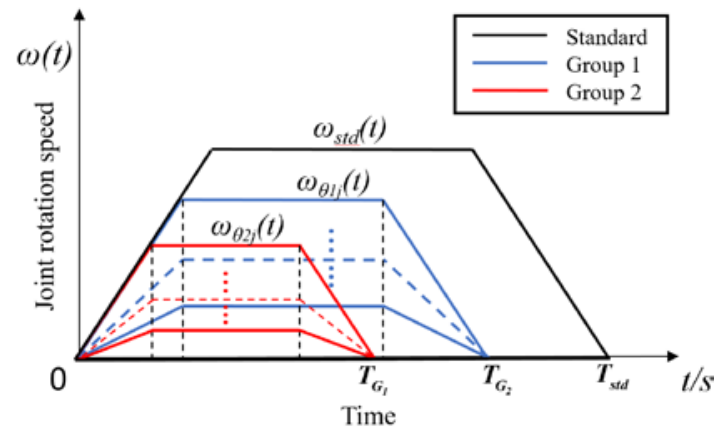

(b)

Fig.3 Multi-joint drive speed coordinated control, (a) Speed-equal proportional coordination (SPC);

(b) Speed-equal time coordination (STC)

(2) Speed-equal time coordination (STC)

From the above calculations, the motion angle velocity curve corresponding to the maximum rotation angle between robot poses can be obtained (Fig. 3(b)). Unified five articulation drive motion acceleration section, constant speed section, decelerating motion time period. The whole motion process shows that the joints complete the acceleration-uniform motion-deceleration motion process at different speeds in the same time interval. Determined robot articulation angular velocity profile.

$$
\omega_{\theta_{i j}}(t)=\left\{\begin{array}{ccc}
\frac{9 \theta_{i j}}{2 T_{G_{i}}{ }^{2}} \cdot t & 0 \leq t \leq \frac{T_{G_{i}}}{3} & \\
\frac{3 \theta_{i j}}{2 T_{G_{i}}} & \frac{T_{G_{i}}}{3}<t \leq \frac{2 T_{G_{i}}}{3} & i=1,2 \\
-\frac{9 \theta_{i j}}{2 T_{G_{i}}{ }^{2}} \cdot t+\frac{9 \theta_{i j}}{2 T_{G_{i}}} & \frac{2 T_{G_{i}}}{3}<t \leq T_{G_{i}} &
\end{array}\right.
$$

The speed function $\omega_{\theta_{i j}}(t)$ and time function $T_{G_{i}}$ obtained by the multi-joint drive speed coordinated control are substituted into the energy function $W_{A}$, and the energy consumption of the gait can be obtained. 


\section{MATLAB simulation analysis}

\subsection{Robot model establishment}

In the MATLAB software simulation analysis robot movement process, first must establish the organization model. Referring to the D-H parameters of the robot, call the Link, Robot, SerialLink functions in the Robotic Toolbox V9.10 to create a model for the robot.

\subsection{Joint drive speed comparison}

The joint rotation angle difference corresponding to different inverse kinematics solutions is substituted into equations (6) and (7). The robot joint angle speed function expression obtained by analytical calculation can be obtained. As shown in Fig.4(a) (b), the speed function curve of the joint rotation speed function curve obtained by the multi-joint drive speed analysis method and the fifth-degree polynomial interpolation method of the robot toolbox is compared.

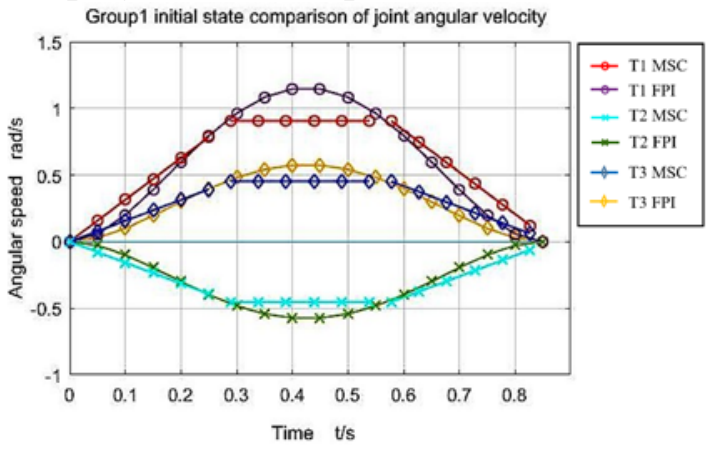

(a)

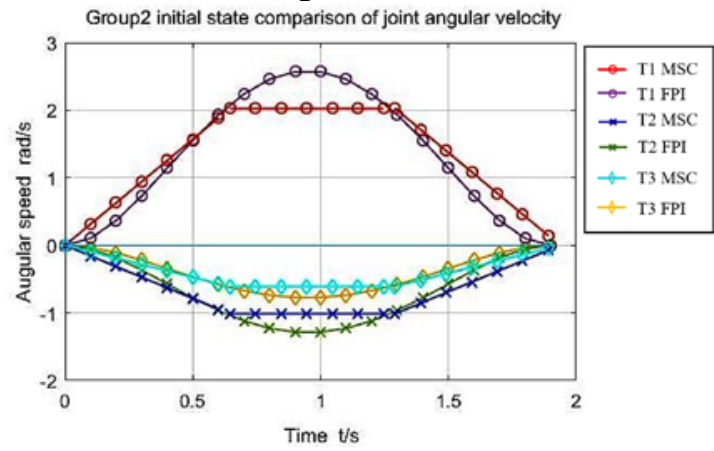

(b)

Fig.4 Joint angular velocity function comparison, (a) Group1 of kinematic inverse solutions; (b) Group2 of kinematic inverse solutions

Obviously, the trapezoidal joint speed function curve obtained by the multi-joint-drive speed coordinated control (MSC) analytical algorithm coincides well with the fifth-order polynomial interpolation method (FPI) simulation speed curve. The rationality and correctness of the joint speed in the gait of this method were verified.

\section{Conclusions}

(1) For the issue of multiple solutions of redundant cascade robot inverse kinematics, the study proposes the multi-joint thin-strip driving energy consumption optimization initially to solve the robot inverse kinematics, with the problem of multiple inverse kinematics optimization solutions solved from the perspective of the lowest energy consumption.

(2) The study uses MATLAB Robotic Toolbox simulation to compare the driving speed function of FPI method simulation and MSCC method, and verifies the correctness and rationality of the multi-joint-drive speed coordinated energy consumption method.

\section{References}

[1] Guan Y, Jiang L, Zhu H, et al. Climbot: A modular bio-inspired biped climbing robot[C]. Ieee/rsj International Conference on Intelligent Robots and Systems. IEEE, 2011:1473-1478.

[2] Guan Y, Zhu H, Wu W, et al. A Modular Biped Wall-Climbing Robot with High Mobility and Manipulating Function [J]. IEEE/ASME Transactions on Mechatronics, 2013, 18(6):1787-1798.

[3] Siciliano B, Sciavicco L, Villani L, et al. Robotics: modelling, planning and control [M]. Springer Publishing Company, Incorporated, 2010.

[4] Lee S, Park J, Han C. Optimal control of a mackerel-mimicking robot for energy efficient trajectory tracking [J]. Journal of Bionic Engineering, 2007, 4(4):209-215.

[5] Saramago S F P, Ceccarelli M. Effect of basic numerical parameters on a path planning of robots taking into account actuating energy[J]. Mechanism \& Machine Theory, 2004, 39(3):247-260.

[6] Dang P, Tan Y F, Shen L M. Control strategy of minimal energy consumption based on multi-joint robot[J]. Application Research of Computers, 2010. 